\title{
Estudio y optimización de los parámetros de reacción para la obtención de material geopolimérico
}

\author{
I. BELEÑA' ${ }^{1}$, M.J.L. TENDERO', E. M. TAMAYO², D. VIE² \\ ${ }^{1}$ Asociación de Investigación de las empresas de la Construcción (AIDICO) \\ ${ }^{2}$ Instituto de Ciencias de Materiales de la Universidad de Valencia
}

\begin{abstract}
La activación alcalina en condiciones de presión y temperatura próximas a las ambientales de algunas arcillas calcinadas da lugar a materiales cementantes de altas prestaciones mecánicas constituidos por redes $3 \mathrm{D}$ polisialato-siloxo, también denominados geopolímeros. En el presente trabajo se ha analizado la influencia de diferentes parámetros de composición $\left(\mathrm{SiO}_{2} / \mathrm{Al}_{2} \mathrm{O}_{3^{\prime}} \mathrm{Na}_{2} \mathrm{O} / \mathrm{Al}_{2} \mathrm{O}_{3}\right.$ y $\left.\mathrm{H}_{2} \mathrm{O} / \mathrm{Na}_{2} \mathrm{O}\right)$, sobre la naturaleza de los materiales formados a partir de metacaolinita y silicato sódico en medio fuertemente alcalino (NaOH) mediante DRX, ${ }^{27} \mathrm{Al}$ y ${ }^{29} \mathrm{Si}-\mathrm{RMN}$ y MEB. Se ha optimizado el proceso de obtención de piezas geopoliméricas variando las condiciones de curado y secado, obteniéndose materiales de características comparables a los cerámicos. El seguimiento de la reacción con el tiempo mediante ${ }^{27} \mathrm{Al}$ y ${ }^{29} \mathrm{Si}-\mathrm{RMN}$ mostró tres etapas bien diferenciadas de disolución, nucleación y polimerización.

Palabras clave: Geopolímero, activación alcalina, polisialato-siloxo, metacaolinita, resistencia a compresión.

\section{Study and obtimizing of the reaction parametres for geopolymeric material manufacture}

The alkali activation of some bulk clays at room pressure and temperature leads to high strength cementitious materials called geopolymers. These inorganic polymers consist of 3D polysialate framework.. Literature concerning this kind of materials is mainly based on patents because of their industrial interest. Influence of several composition parameters $\left(\mathrm{SiO}_{2} / \mathrm{Al}_{2} \mathrm{O}_{3}, \mathrm{Na}_{2} \mathrm{O} / \mathrm{Al}_{2} \mathrm{O}_{3}\right.$ y $\left.\mathrm{H}_{2} \mathrm{O} / \mathrm{Na}_{2} \mathrm{O}\right)$ in the structure and properties of geopolymeric materials, synthesised from metakaolinite and sodium silicate in a highly alkaline environment, has been studied by XRD, ${ }^{27} \mathrm{Al} \mathrm{y}{ }^{29} \mathrm{Si}-\mathrm{NMR}$ and MES. Process for the obtention of geopolymeric pieces has been optimised varying the cured and drying conditions, obtaining likeceramic products. The different geopolymerization reaction periods have been followed by ${ }^{27} \mathrm{Al} \mathrm{y}{ }^{29} \mathrm{Si}-\mathrm{NMR}$.
\end{abstract}

Keywords: Geopolymer, alkali activation, polysilicate, metakaolinite, compression resistance

\section{INTRODUCCIÓN}

Basándose en el hecho de que el método de obtención de algunos plásticos fenólicos y zeolitas se desarrollaban en condiciones muy similares (hidrotermales), científicos franceses sintetizaron los primeros geopolímeros [1-3]. Estos son polímeros inorgánicos formados por redes $3 \mathrm{D}$ amorfas de tetraedros alternados de $\mathrm{AlO}_{4}^{-}$y $\mathrm{SiO}_{4}$ de gran estabilidad térmica y química, no inflamables, con buenas prestaciones mecánicas y fácilmente procesables a temperaturas inferiores a $100{ }^{\circ} \mathrm{C}$ y presión atmosférica. Hasta el momento la mayoría de la bibliografía existente se encuentra en forma de patentes por su interés industrial y hay muy pocos estudios sobre la influencia de los distintos parámetros de reacción en la naturaleza y propiedades de los materiales geopoliméricos [4-7], así como el mecanismo y cinética de reacción [8]. Siguiendo el método de obtención propuesto por Davidovits se ha analizado mediante DRX, ${ }^{27} \mathrm{Al}$ y ${ }^{29} \mathrm{Si}-\mathrm{RMN}$ y MEB la influencia de la composición y condiciones de obtención en estructura y microestructura de la red geopolimérica. Además se ha optimizado el proceso de obtención para producir materiales geopoliméricos de características similares a los cerámicos y adecuado para la fabricación de baldosas. Por último, se ha realizado el seguimiento de la reacción geopolimérica, en condiciones ambientales, mediante ${ }^{27} \mathrm{Al}$ y ${ }^{29} \mathrm{Si}-\mathrm{RMN}$ que ha permitido determinar los tiempos en que transcurren las distintas fases de reacción.

\section{PARTE EXPERIMENTAL}

\subsection{Materiales de partida}

En la preparación de los geopolímeros se partió de caolinita natural (Sigma \& Aldrich Química S.A), Aerosil 200 (Degussa- Hüls), As- trosil 116 (Massó y Carol, S.A.) e Hidróxido Sódico P.A. en lentejas 98\% (Prolabo, S.A) y agua destilada. En la tabla I se muestra la composición en $\%$ peso de óxidos metálicos de todos los materiales de partida y sus características físicas.

TABLA I.- COMPOSICIÓN POR ÓXIDOS MINERALES DE LOS PRODUCTOS DE PARTIDA

\begin{tabular}{|l|c|c|c|c|c|c|c|c|c|c|}
\hline \% óxidos & $\mathrm{SiO}_{2}$ & $\mathrm{Al}_{2} \mathrm{O}_{3}$ & $\mathrm{Fe}_{2} \mathrm{O}_{3}$ & $\mathrm{Na}_{2} \mathrm{O}$ & $\mathrm{K}_{2} \mathrm{O}$ & $\mathrm{TiO}_{2}$ & $\mathrm{P.C}^{*}$ & $\mathrm{~d}_{50}{ }^{* *}(\lambda \mathrm{m})$ & $\mathrm{d}\left(\mathrm{g} / \mathrm{cm}^{3}\right)$ & $\mathrm{BET}^{* * *}\left(\mathrm{~m}^{2} / \mathrm{g}\right)$ \\
\hline Caolinita & 54.86 & 30.86 & 0.5 & 0.1 & 1.8 & 0.4 & 12.2 & 13.48 & 2.6 & 9.0 \\
\hline Aerosil & 99.8 & 0.05 & 0.003 & 0.0 & 0.0 & 0.03 & 0.12 & 32.23 & 2.2 & 200.0 \\
\hline $\mathrm{Na}_{2} \mathrm{SiO}_{4} \times \mathrm{xH}_{2} \mathrm{O}$ & 27.6 & 0.0 & 0.0 & 17.4 & 0.0 & 0.0 & 55.0 & -- & $1.55-1.59$ & --- \\
\hline
\end{tabular}

${ }^{*}$ P.C. Pérdida de masa por calcinación

${ }^{* *} \mathrm{~d}_{50}:$ Tamaño medio de partícula

${ }^{* * *}$ BET: Area superficial

\subsection{Caracterización microscópica y macroscópica}

Los registros de Difracción de Rayos X de polvo desorientado se han realizado en un equipo PHILIPS PW-1700 con un monocromador de grafito y usando radiación CuK. Los espectros de difracción de RMN se adquirieron utilizado un equipo Varian $300 \mathrm{MHz}$ con una Sonda Multinuclear para sólidos CP/MAS. Los espectros de ${ }^{29} \mathrm{Si}$ se han adquirido a $59.59 \mathrm{MHz}$, tomando como referencia el tetrametilsilano (TMS) y los de ${ }^{27} \mathrm{Al}$ a $78.17 \mathrm{MHz}$, tomando de referencia el $\mathrm{Al}\left(\mathrm{H}_{2} \mathrm{O}\right)_{6}{ }^{3+}$. Se observó la posible existencia de defectos (grietas o poros, manchas, aspecto de la superficial) y midió la dureza Vickers y la resistencia a compresión (Norma UNE-80.101) de las piezas procesadas. 


\section{RESULTADOS Y DISCUSIÓN}

3.1. Influencia de los parámetros de composición $\mathrm{Si}_{2} \mathrm{O} / \mathrm{Al}_{2} \mathrm{O}_{3}$ $\mathrm{Na}_{2} \mathrm{O} / \mathrm{Al}_{2} \mathrm{O}$ y $\mathrm{H}_{2} \mathrm{O} / \mathrm{Na}_{2} \mathrm{O}$ en la estructura de la red geopolimérica

En todas las muestras analizadas por DRX se observó la existencia de una banda ancha entre $20-40^{\circ} 2 \theta$, correspondiente a la fase amorfa característica de los geopolímeros [10], así como los picos correspondientes a la Moscovita y el Cuarzo de la metacaolinita de partida. Se observaron dos tipos de muestras de distinta naturaleza (ver figura 1) en unas predominan las fases cristalinas zeolíticas y en otras la fase amorfa geopolimérica. En el primer caso, la formación de zeolitas tiene lugar siempre que se da una relación $\mathrm{SiO}_{2} / \mathrm{Al}_{2} \mathrm{O}_{3}=3$, y un exceso de $\mathrm{Na}^{+}$respecto al $\mathrm{AlO}_{2}^{-}\left(\mathrm{Na}_{2} \mathrm{O} / \mathrm{Al}_{2} \mathrm{O}_{3}>1\right)$. Este exceso de ión alcalino favorece además la formación de carbonatos alcalinos que inhiben la reacción de geopolimerización. Relaciones $\mathrm{Si}_{2} \mathrm{O} / \mathrm{Al}_{2} \mathrm{O}_{3} \geq 4$ y $\mathrm{M}_{2} \mathrm{O} / \mathrm{Al}_{2} \mathrm{O}_{3}$ $\approx 1$ favorecen la formación de muestras de naturaleza predominantemente amorfa.
El espectro de ${ }^{27} \mathrm{Al}-\mathrm{RMN}$ mostrado en la figura 2 muestra la evolución de los entornos de los núcleos de $\mathrm{Al}$ en función de los parámetros de composición. Se observa en todos los casos, una única banda característica de núcleos de $\mathrm{Al}(\mathrm{IV})$. Los espectros de muestras con naturaleza zeolítica presentan bandas más estrechas y desplazadas hacia frecuencias mayores $(60 \mathrm{ppm})$ que aquellas muestras de naturaleza amorfa (50-55 ppm). En general, se observó un desplazamiento de los máximos de las bandas hacia frecuencias menores al aumentar la relación $\mathrm{SiO}_{2} / \mathrm{Al}_{2} \mathrm{O}_{3}$, debido a la influencia de los núcleos de la segunda esfera de coordinación del Al. En la figura 2 también se muestra la evolución del entorno del Si mediante ${ }^{29} \mathrm{Si}-\mathrm{RMN}$. El máximo de las bandas de los compuestos se van desplazando hacia frecuencias de resonancia menores al aumentar la relación $\mathrm{SiO}_{2} / \mathrm{Al}_{2} \mathrm{O}_{3}$. Esto puede ser debido a que los entornos de tipo $\mathrm{SiQ}^{4}(0 \mathrm{Al})$ aumentan en detrimento de los de tipo $\mathrm{SiQ}^{4}(4 \mathrm{Al})$. Por otro lado, se observa como cuando la relación $\mathrm{NaO}_{2} / \mathrm{Al}_{2} \mathrm{O}_{3}$ es superior a 1 influye en el desplazamiento químico de los núcleos de ${ }^{29} \mathrm{Si}$ y ${ }^{27} \mathrm{Al}$ debido posiblemente a que, una vez compensada la carga negativa asociada al $\mathrm{Al}$ tetracoordinado, un aumento de la concentración de cationes $\mathrm{Na}^{+}$aumenta las inte-

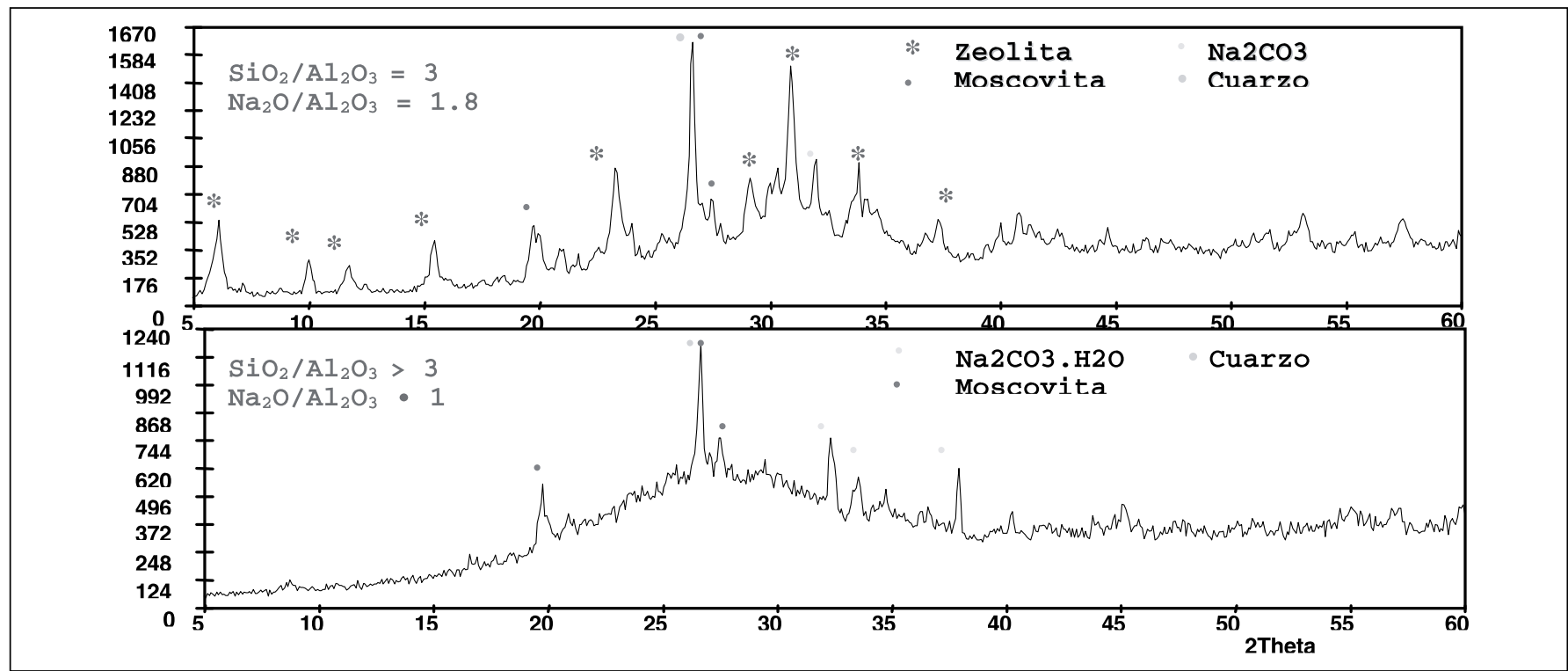

Figura 1.-Espectros de DRX de los dos tipos de muestras. A) predominio de fases zeolíticas. B) predominio de fase amorfa geopolimérica.

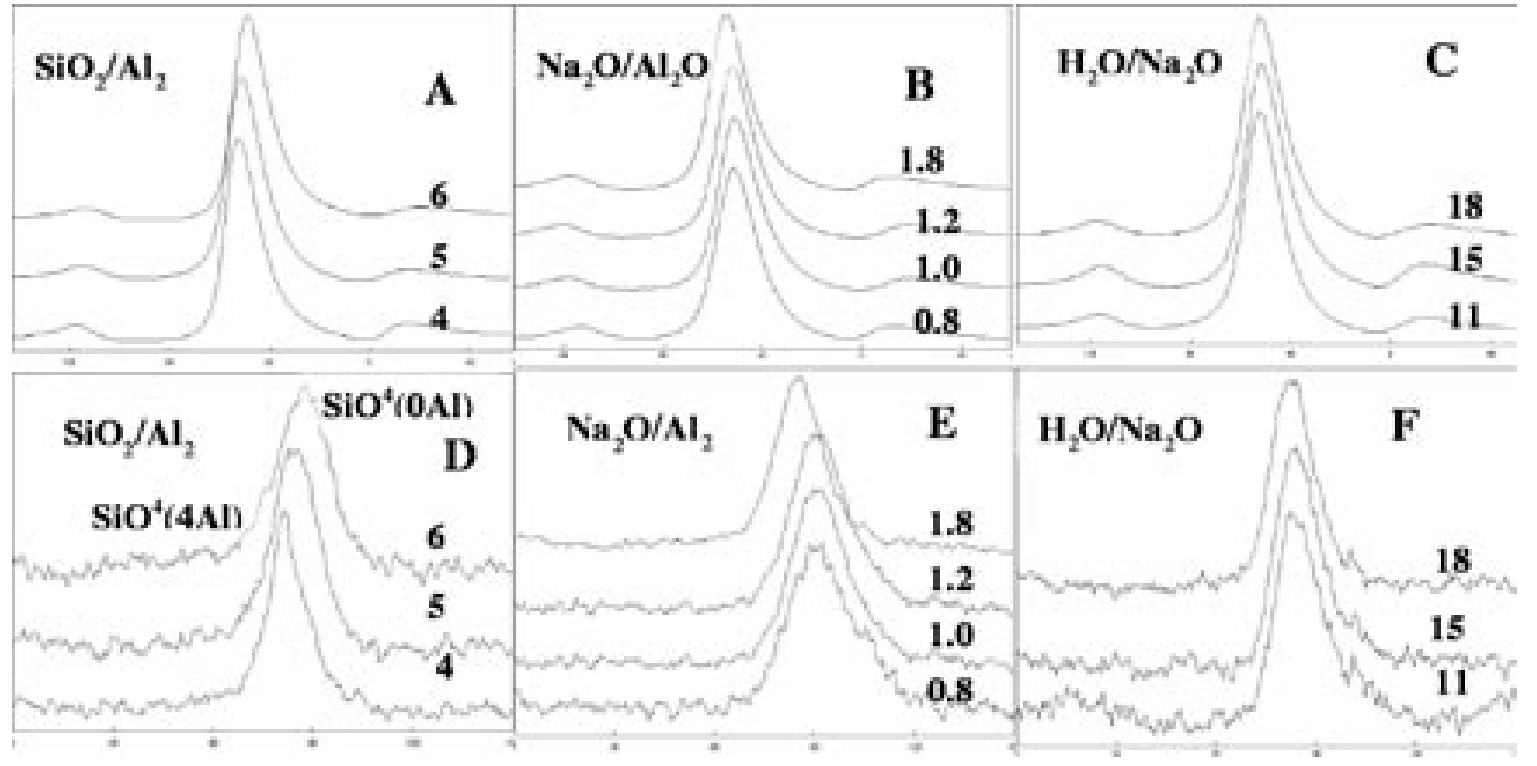

Figura 2.- Estudio de la influencia de los parámetros de composición: A y D) $\mathrm{SiO}_{2} / \mathrm{Al}_{2} \mathrm{O}_{3}$ B y E) $\mathrm{Na}_{2} \mathrm{O} / \mathrm{Al}_{2} \mathrm{O}_{3^{\prime}} \mathrm{C}$ y F) $\mathrm{H}_{2} \mathrm{O} / \mathrm{Na}_{2} \mathrm{O}$ 
racciones electrostáticas entre ellos, desestabilizando la red 3D y por tanto afectando a los niveles nucleares de energía de los átomos que la constituyen. En cuanto a la relación $\mathrm{H}_{2} \mathrm{O} / \mathrm{Na}_{2} \mathrm{O}$, no parece afectar significativamente ni al entorno local del $\mathrm{Al}$, ni al del $\mathrm{Si}$ en las muestras de naturaleza amorfa.

3.3. Optimización de los parámetros de composición $\mathrm{Si}_{2} \mathrm{O} /$ $\mathrm{Al}_{2} \mathrm{O}_{3}, \mathrm{Na}_{2} \mathrm{O} / \mathrm{Al}_{2} \mathrm{O}$ y $\mathrm{H}_{2} \mathrm{O} / \mathrm{Na}_{2} \mathrm{O}$ y condiciones de curado y secado

En la tabla II aparecen las formulaciones que dieron lugar a resistencias a compresión más altas. Se observa como el método de curado-secado a temperatura ambiente produce resistencias superiores, posiblemente debido a que la deshidratación y deshidroxilación a $40^{\circ} \mathrm{C}$ sea demasiado rápida ocasionando la formación de microfisuras en las piezas. Las formulaciones que dieron mejores resistencias a compresión coinciden con las de aquellas muestras de naturaleza amorfa $\left(\mathrm{SiO}_{2} / \mathrm{Al}_{2} \mathrm{O}_{3}\right.$ entre 4 y 5, $\mathrm{Na}_{2} \mathrm{O} / \mathrm{Al}_{2} \mathrm{O}_{3}$ igual a 1.0). La relación $\mathrm{H}_{2} \mathrm{O} / \mathrm{Al}_{2} \mathrm{O}_{3}$ de 11 dio valores de resistencia superiores a aquellas con mayor porcentaje de agua, lo que puede indicar que un exceso de agua puede favorecer la formación de carbonatos alcalinos que afectan a la resistencia mecánica de las muestras

TABLA II. FORMULACIONES DE LAS MUESTRAS QUE PRESENTARON RESISTENCIAS A COMPRESIÓN SUPERIOR.

\begin{tabular}{|l|c|c|c|c|c|}
\hline \multicolumn{1}{|c|}{ Ref } & $\mathbf{S i O}_{2} / \mathbf{A l}_{2} \mathbf{O}_{3}$ & $\mathbf{N a}_{2} \mathbf{O} / \mathbf{A l}_{2} \mathbf{O}_{3}$ & $\mathbf{H}_{2} \mathbf{O} / \mathbf{N a}_{\mathbf{2}} \mathbf{O}$ & $\mathbf{R c}^{1}(\mathbf{M P a})$ & $\mathbf{R c}^{2}(\mathbf{M P a})$ \\
\hline $\mathrm{MkNa} 2$ & 4 & 0.8 & 11 & - & 61.9 \\
\hline $\mathrm{MkNa6}$ & 5 & 1.0 & 11 & - & 70.4 \\
\hline $\mathrm{MkNa} 9$ & 5 & 1.2 & 11 & 45.3 & 56.6 \\
\hline $\mathrm{MkNa} 15$ & 4 & 0.8 & 15 & 43.9 & 41.8 \\
\hline $\mathrm{MkNa} 19$ & 5 & 1.0 & 15 & 15.9 & 46.8 \\
\hline $\mathrm{MkNa} 22$ & 5 & 1.2 & 15 & 40.3 & 28.7 \\
\hline $\mathrm{MkNa} 28$ & 4 & 0.8 & 18 & 9.4 & 29.4 \\
\hline MkNa32 & 5 & 1.0 & 18 & - & 30.2 \\
\hline
\end{tabular}

\subsection{Estudio de la cinética de la reacción de polimerización por ${ }^{27} \mathrm{Al}$ y ${ }^{29} \mathrm{Si}-\mathrm{RMN}$}

Se hizo un estudio de la evolución de los entornos de Al y Si desde el momento en realizarse la mezcla de los productos de partida hasta pasados varios días. En la figura 3 se muestran los espectros de ${ }^{27} \mathrm{Al}$ y ${ }^{29} \mathrm{Si}-\mathrm{RMN}$ para el caso concreto de la muestra MkNa15 junto con los materiales de partida. En el seguimiento por ${ }^{27} \mathrm{Al}-\mathrm{RMN}$, se aprecia como durante las dos primeras horas, los $\mathrm{Al}(\mathrm{V})$ y $(\mathrm{VI})$ se transforman en $\mathrm{Al}(\mathrm{IV})$ hasta su desaparición. También puede apreciarse un desplazamiento del máximo de la banda de $\mathrm{Al}(\mathrm{IV})$ durante el proceso de curado. Durante la primera hora se observa un desplazamiento de la banda hacia frecuencias mayores, que puede indicar la disolución de la metacaolinita. En la siguiente hora se produce el periodo de inducción o nucleación, durante el cual no se aprecia prácticamente ningún cambio significativo en la posición de la banda. A partir de ese tiempo la banda de $\mathrm{Al}(\mathrm{IV})$ comienza a desplazarse hacia frecuencias inferiores, indicando que se está produciendo la polimerización. Inicialmente se forman cadenas sencillas (entorno $\mathrm{AlQ}^{2}(2 \mathrm{Si})$ ) que se van entrecruzando hasta formar redes tridimensionales amorfas (entorno $\mathrm{AlQ}^{4}(4 \mathrm{Si})$ ). Esta etapa se prolonga hasta un tiempo de $24 \mathrm{~h}$, durante el cual la banda alcanza la posición de menor frecuencia. A partir de entonces no se observa ninguna evolución de la misma. En el seguimiento mediante ${ }^{29} \mathrm{Si}-\mathrm{RMN}$ de la misma muestra se observa como las materias primas se disuelven $\left(\mathrm{Q}^{0}\right)$ dando lugar a iones Silicato y Aluminato que van condensando y formando especies sencillas $\left(\mathrm{Q}^{1}\right)$ que por polimerización

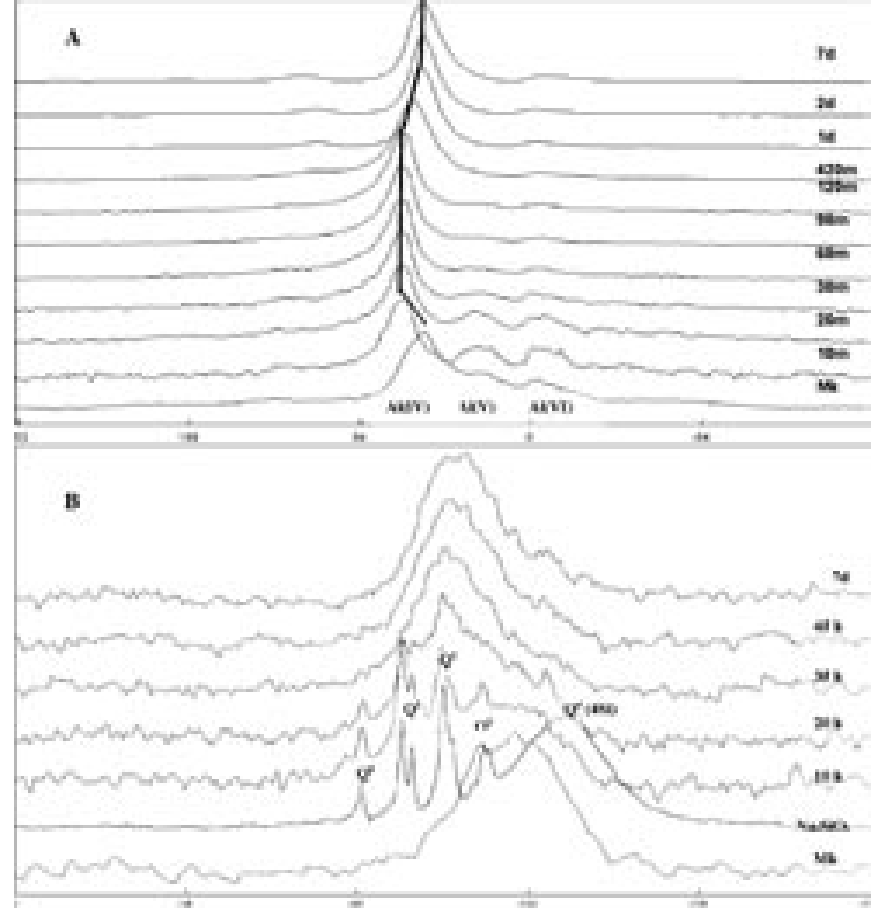

Figura 3.- A) Seguimiento de la reacción de geopolimerización por A) ${ }^{27} \mathrm{Al}-\mathrm{RMN}$ y por B) ${ }^{29} \mathrm{Si}-\mathrm{RMN}$

dan lugar a cadenas $\left(Q^{2}\right)$ y éstas a su vez por entrecruzamiento a redes tridimensionales amorfas $\left(\mathrm{Q}^{3}\right.$ y $\left.\mathrm{Q}^{4}\right)$. A las $10 \mathrm{~h}$ se puede observar la existencia todavía de estas especies junto con un entorno $Q^{4}$ indicativo de que la reacción de polimerización ha dado comienzo. A partir de las 35 horas no se aprecia prácticamente evolución del espectro, por lo que la reacción concluye entre las 20 y 35 horas de acuerdo también con los resultados obtenidos mediante ${ }^{27} \mathrm{Al}-\mathrm{RMN}$.

\section{CONCLUSIONES}

El control de los parámetros de composición y curado permite diseñar materiales de distinta naturaleza y propiedades. Relaciones $\mathrm{Si}_{2} \mathrm{O} / \mathrm{Al}_{2} \mathrm{O}_{3}$ bajas y exceso de catión $\mathrm{Na}^{+}$respecto al $\mathrm{Al}(\mathrm{IV})$, favorecen la obtención de zeolitas de bajas prestaciones mecánicas. Por el contrario, relaciones $\mathrm{Si}_{2} \mathrm{O} / \mathrm{Al}_{2} \mathrm{O}_{3} \geq 4$ y $\mathrm{M}_{2} \mathrm{O} / \mathrm{Al}_{2} \mathrm{O}_{3}=1$ y condiciones de curado suaves (temperatura y presión ambiente) favorecen la obtención de materiales de estructura amorfa y naturaleza geopolimérica de dureza y resistencia mecánicas similar a los materiales cerámicos.

Cuando la relación $\mathrm{Na}_{2} \mathrm{O} / \mathrm{Al}_{2} \mathrm{O}_{3}>1$, un aumento en la concentración de cationes $\mathrm{Na}^{+}$altera los niveles nucleares de energía de los átomos que conforman la red 3D y la desestabiliza, disminuyendo la resistencia mecánica. En cuanto a la relación $\mathrm{H}_{2} \mathrm{O} / \mathrm{Na}_{2} \mathrm{O}$, no afecta significativamente al entorno local del $\mathrm{Al}$ y $\mathrm{Si}$ en las muestras de naturaleza amorfa. Sin embargo, una excesiva cantidad de agua también puede favorecer la difusión de los iones $\mathrm{Na}^{+}$hacia la superficie, inhibiendo su papel como catalizador compensador de carga y estabilizador de la red geopolimérica.

Durante la primera hora se produce la disolución de los materiales de partida, seguido de un periodo de inducción o nucleación en el cual no se aprecian prácticamente cambios en la evolución de los espectros. A las dos horas comienza la etapa de curado o polimerización de la muestra durante la cual se forman cadenas sencillas inicialmente que se van entrecruzando hasta formar redes tridimensionales amorfas. Esta etapa se prolonga hasta un tiempo de $24 \mathrm{~h}$. 


\section{BIBLIOGRAFÍA}

1. J. Davidovits, "Geopolymers: inorganic polymers new materials", J. Thermal Analysis, Vol. 37, pp. 1633-1656 (1991).

2. J. Davidovits, Chemistry of geopolymeric systems, terminology, Geopolymer 99', Vol. 1, pp.9, Saint Quentin, France, 1999.

3. Hua Xu, J.S.J. Van Deventer, The geopolymerisation of natural alumino-silicates, Geopolymer '99 Procedings, Saint Quentin, France, 1999.

4. J. Davidovits, Structural characterization of geopolymeric materials with X-Ray diffractometry and MAS-NMR spectroscopy, Geopolymer 88', Vol. 2, pp. 149-166, Saint Quentin, France, 1998.

5. J.G.S. Van Jaarsveld,. J.S.J. Van Deventer, Effect of the alkali metal activator on the properties of fly ash-based geopolymers. Ind. Eng. Chem. Res. 1999 $38,3932-3941$.
6. V. Barbosa, Synthesis and characterisation of materials based on inorganic polymers of alumina and silica: sodium polysialates polymers, The Int. Journal of Inorg. Mat. 2000, 2:4:309-317

7. M.L. Granizo, M.T. Blanco. Alkali Activation of metakaolin. An isotherma conduction calorimetry study, Journal of Thermal Analysis, Vol. 52 (1998) 957-965.

8. S. Alonso, A. Palomo, Alkaline activation of metakaolín and calcium hydroxide mixtures: influence of temperature, activator concentration and solids ratio, Materials Letters 2001, 47: 1-2:55-62.

9. J. Davidovits, Mineral Polymers and Methods of Making Them. US Patent $4,349,386$

10. J. Davidovits, Chemistry of geopolymeric systems, terminology. GeoPolymer'99, Saint Quentin, France, 1999.

Recibido: 1.2 .03

Aceptado: 30.11 .03 